\title{
BMJ Open Crizanlizumab and comparators for adults with sickle cell disease: a systematic review and network meta- analysis
}

\author{
Howard Thom, ${ }^{1}$ Jeroen Jansen, ${ }^{2}$ Jason Shafrin, ${ }^{2}$ Lauren Zhao (D) , \\ George Joseph, ${ }^{3}$ Hung-Yuan Cheng, ${ }^{1}$ Subhajit Gupta, ${ }^{3}$ Nirmish Shah ${ }^{4}$
}

To cite: Thom H, Jansen J, Shafrin J, et al. Crizanlizumab and comparators for adults with sickle cell disease: a systematic review and network meta-analysis. BMJ Open 2020;10:e034147. doi:10.1136/ bmjopen-2019-034147

- Prepublication history and supplemental information for this paper are available online. To view these files, please visit the journal online (http://dx.doi. org/10.1136/bmjopen-2019034147).

Received 09 September 2019 Revised 20 April 2020 Accepted 14 July 2020

Check for updates

(c) Author(s) (or their employer(s)) 2020. Re-use permitted under CC BY-NC. No commercial re-use. See rights and permissions. Published by BMJ.

${ }^{1}$ Bristol Medical School, University of Bristol, Bristol, UK ${ }^{2}$ Health Economics,

PRECISIONheor, Los Angeles, California, USA

${ }^{3}$ Novartis Pharmaceuticals Corp, East Hanover, New Jersey, USA

${ }^{4}$ Department of Medicine,

Duke University, Durham, North Carolina, USA

Correspondence to

Dr Howard Thom;

howard.thom@bristol.ac.uk

\section{ABSTRACT}

Objectives Treatment options for preventing vasoocclusive crises (VOC) among patients with sickle cell disease (SCD) are limited, especially if hydroxyurea treatment has failed or is contraindicated. A systematic literature review (SLR) and network meta-analysis (NMA) were conducted to evaluate the efficacy and safety of crizanlizumab for older adolescent and adult ( $\geq 16$ years old) SCD patients.

Methods The SLR included randomised controlled trials (RCTs) and uncontrolled studies. Bayesian NMA of VOC, all-cause hospitalisation days and adverse events were conducted.

Results The SLR identified 51 studies and 9 RCTs on 14 treatments that met the NMA inclusion criteria. The NMA found that crizanlizumab $5.0 \mathrm{mg} / \mathrm{kg}$ was associated with a reduction in VOC (HR 0.55, 95\% credible interval $(0.43$, 0.69 ); Bayesian probability of superiority $>0.99$ ), all-cause hospitalisation days $(0.58(0.50,0.68) ;>0.99)$ and no evidence of difference on adverse events $(0.91(0.59,1.43)$ $0.66)$ or serious adverse events $(0.93(0.47,1.87) ; 0.59)$ compared with placebo. The HR for reduction in VOC for crizanlizumab relative to L-glutamine was $(0.67(0.50$, $0.88) ;>0.99$ ). These results were sensitive to assumptions regarding whether patient age is an effect modifier. Conclusions This NMA provides preliminary evidence comparing the efficacy of crizanlizumab with other treatments for VOC prevention.

\section{INTRODUCTION}

Sickle cell disease (SCD) affects approximately 100000 people in the USA. ${ }^{1}$ The disease is caused by an autosomal-recessive single gene defect in the beta chain of haemoglobin, which results in sickle cell haemoglobin. Sickled cells break down prematurely, and are associated with varying degrees of anaemia. Interactions of red blood cells, white blood cells, platelets and endothelial cells are an important contributor to the pathophysiology of SCD..$^{2-7}$ For instance, endothelial cells lining the vasculature are activated and have increased expression of adhesion molecules in SCD patients; this plays a central role
Strengths and limitations of this study

- This systematic literature review was comprehensive in terms of outcomes and interventions and was focused on the target population of crizanlizumab.

- To include a diverse range of outcome summaries, a shared parameter Bayesian network meta-analysis was employed, as recommended by National Institute for Health and Care Excellence.

- Risk of bias was assessed using the best practice Cochrane collaboration tool.

- It was not possible to adjust for differences in statistical analysis across randomised controlled trials.

- The strength of comparisons on outcomes other than vaso-occlusive crises (VOC) was weak, and VOC may not be the key outcome for patients.

in the development of vaso-occlusion. ${ }^{3} 89$ Ultimately, obstruction of small blood capillaries causes painful crises, damage to major organs and increased vulnerability to severe infections. Over the past several decades, life expectancy has improved, however, the disease continues to be associated with early mortality and high morbidity. ${ }^{10}$ The aim of treatment is to aid disease and chronic pain management, reduce severity and/or prevent complications and manage acute pain during crises. ${ }^{11}$

There is no widely available cure for SCD and few effective treatments. Hydroxyurea and L-glutamine (Endari), the only two Food and Drug Administration (FDA)-approved drugs for SCD, are indicated for the prevention of vaso-occlusive crises (VOC). ${ }^{12}$ In a 2-year paediatric study, per patient healthcare costs for children on hydroxyurea were $\$ 9450$, compared with $\$ 13716$ for those who did not receive this treatment. ${ }^{13}$ Despite the National Heart, Lung and Blood Institute's recommendations, hydroxyurea is not regularly prescribed and adherence to the therapy 
is poor. ${ }^{14}$ Further, there are no current clinical guidelines outlining when to integrate L-glutamine into care. Regular blood transfusions can also be used as a preventive measure, but they may also lead to abnormally high levels of iron in the blood, which can cause long-term organ damage and reactions due to a mismatch between the donors and recipients. ${ }^{14}$ Voxelotor has shown an ability to increase haemoglobin levels in patients with SCD $^{15}$ and in November 2019 was FDA-approved. ${ }^{16}$

Crizanlizumab is a new, FDA-approved ${ }^{17}$ drug for the prevention of VOC. A phase II multicentre, randomised, placebo-controlled, double-blind, 12-month study was completed to evaluate crizanlizumab $5.0 \mathrm{mg} / \mathrm{kg}$ and $2.5 \mathrm{mg} / \mathrm{kg}$ versus placebo. ${ }^{18}$ This study found that the median rate of crises per year was 1.63 with crizanlizumab $5.0 \mathrm{mg} / \mathrm{kg}$ versus $2.98 \mathrm{mg} / \mathrm{kg}$ with placebo (indicating a $45.3 \%$ lower rate with high-dose crizanlizumab $5.0 \mathrm{mg}$ / $\mathrm{kg}, \mathrm{p}=0.01$ ). The median time to the first VOC was also significantly longer with high-dose crizanlizumab $5.0 \mathrm{mg} /$ $\mathrm{kg}$ than with placebo ( 4.07 vs 1.38 months, $\mathrm{p}=0.001$ ), as was the median time to the second VOC (10.32 vs 5.09 months, $\mathrm{p}=0.02)$. In addition, the median rate of uncomplicated crises per year was 1.08 with crizanlizumab $5.0 \mathrm{mg} / \mathrm{kg}$, as compared with 2.91 with placebo (indicating a $62.9 \%$ lower rate with crizanlizumab $5.0 \mathrm{mg} / \mathrm{kg}$, $\mathrm{p}=0.02)$.

The comparative efficacy and safety of crizanlizumab has been evaluated against placebo, however, head-to-head randomised controlled trial (RCT) evidence is lacking for comparisons to treatments of interest. Network metaanalysis (NMA) is a statistical method that allows for the simultaneous evaluation of all treatments within a therapeutic area and allows for indirect comparisons between treatments where head-to-head evidence may not be available. Specifically, NMA can be used to combine direct and indirect evidence regarding any interventions that form a network of RCTs where each trial has at least one intervention (active or placebo) in common with another trial and all RCTs are sufficiently similar. ${ }^{19}{ }^{20}$ To minimise risk of bias, RCTs should be identified through a comprehensive systematic literature review (SLR) using pre-defined criteria. $^{21}$

This study conducts an SLR and NMA to assess the comparative efficacy and safety of crizanlizumab against relevant competing interventions for older adolescent and adult ( $\geq 16$ years old) patients with SCD.

\section{METHODS}

\section{Systematic literature reviews}

The SLR protocol was finalised on 25 June 2018 and the SLR was conducted according to Preferred Reporting Items for Systematic Reviews and Meta-Analyses (PRISMA) guidelines. ${ }^{22}$ A PRISMA NMA checklist can be found in online supplemental appendix A. The SLR approach updated and expanded an earlier published SLR by Sins $e t a^{23}$ by including non-controlled studies and included additional interventions. Inclusion and exclusion criteria for studies are summarised in table 1. Relevant studies were identified by searching the following databases: Cochrane Central Register of Controlled Trials (CENTRAL); Medical Literature Analysis and Retrieval

Table 1 Study selection criteria to identify trials for the systematic literature review

\begin{tabular}{|c|c|}
\hline Criteria & Description \\
\hline Interventions & $\begin{array}{l}\text { Crizanlizumab } \\
\text { L-glutamine } \\
\text { Voxelotor (GBT440) } \\
\text { Red blood cell transfusions } \\
\text { Other types of transfusions } \\
\text { Any pharmacological interventions for preventing crisis, pain and/or vaso-occlusive crisis } \\
\text { (VOC) }\end{array}$ \\
\hline Comparators & $\begin{array}{l}\text { Placebo or best supportive care } \\
\text { Any of the listed interventions of interest } \\
\text { Any treatment that facilitates an anchored indirect comparison }\end{array}$ \\
\hline Outcomes & $\begin{array}{l}\text { Primary outcome: } \\
\text { Pain, crisis and VOC (frequency, intensity and duration in one event) } \\
\text { Secondary outcomes: } \\
\text { Hospital admission, including emergency department and nurse visits } \\
\text { SCD complications, including acute chest syndromes } \\
\text { Analgesic use } \\
\text { Adverse events* }\end{array}$ \\
\hline Study design & $\begin{array}{l}\text { Randomised controlled trials (RCTs) } \\
\text { Single-arm trials when RCTs are not available for the interventions of interest }\end{array}$ \\
\hline Language & English \\
\hline
\end{tabular}

*In addition to efficacy outcomes, adverse events are of interest for the review, but will not be used as study selection criteria. 
System Online (MEDLINE) and Excerpta Medica database (Embase). We also searched a trial registry, ClinicalTrials.gov. The search strategies were derived from Sins $e t$ $a l^{23}$ and can be found in online supplemental appendix $\mathrm{B}$ along with the complete search protocols in online supplemental appendices $\mathrm{C}$ and $\mathrm{D}$. As blood transfusion was not included by Sins $e t a l,{ }^{23}$ we conducted a separate search for blood transfusion from inception of databases to 30 August 2018. For non-transfusion studies, the search date was from 1 January 2017 to 21 June 2018 to bridge the findings of Sins et al. ${ }^{23}$

Results of searches were managed using Endnote and a Microsoft Excel spreadsheet. Two reviewers screened and selected records independently against inclusion and exclusion criteria using titles and abstracts. Full texts of potential eligible records were retrieved and screened to assess the eligibility for data extraction. Disagreements were resolved by discussion and consensus. Following reconciliation between the two investigators, a third investigator was included to reach consensus for any remaining discrepancies. The Cochrane collaboration's risk of bias tool was used to assess risk of bias in included RCTs. ${ }^{24}$ The Newcastle-Ottawa Scale was used to assess the quality of non-controlled studies. ${ }^{25}$

The primary outcome of this review was sickle cell pain crisis, also known as a VOC leading to a healthcare visit. A variety of definitions for VOC was observed in the included studies. We consulted several medical experts and chose the definition of VOC used in the pivotal phase II RCT of crizanlizumab. ${ }^{18}$ In this trial, a VOC was defined as an acute episode of pain, with no medically determined cause other than a vaso-occlusive event that resulted in a medical facility visit and treatment with oral or parenteral opioids or with a parenteral non-steroidal anti-inflammatory drug. In addition to outcomes specifically named as VOC, the outcomes of pain crisis and SCD crisis were extracted and included with the VOC set if found to use a comparable definition.

Other outcomes identified as of interest and/or extracted included pain-related outcomes, acute chest syndrome, all-cause hospitalisations, transfusions, analgesic use, death, adverse events and serious adverse events. In addition to study and intervention characteristics, the patient characteristics were extracted to qualitatively assess comparability of different study populations.

\section{Network meta-analysis}

This paper adopts the Bayesian statistical framework to conduct the NMA. This is different to the frequentist framework as the data, represented as a likelihood, are used to update a prior distribution on uncertain parameters to provide a posterior distribution. ${ }^{26}$ Bayesian NMA is conducted using Markov chain Monte Carlo (MCMC) estimation which is a technique to sample from the posterior distribution of a specified likelihood and prior. The Bayesian framework is recommended by National Institute for Health and Care Excellence (NICE) and published textbooks for NMA due to its flexibility and in this study it allows the synthesis of different data types, which would be difficult in the frequentist setting. ${ }^{27} 28$ The key outputs of a Bayesian analysis are $95 \%$ credible intervals (CrI) and Bayesian probabilities. The $95 \% \mathrm{CrI}$ is the 95th percentile of the MCMC samples from the posterior distribution and represents a region where there is $95 \%$ probability of containing the true value of some parameter, for example, an HR. The Bayesian probability for a parameter is the proportion of the MCMC distribution that lies above or below a certain threshold; in this analysis, the interest lies in Bayeisan probabilities of superiority which are the probability that the HRs are $>1$.

Quantitative synthesis through this Bayesian NMA approach was planned for reported or derived time-toevent outcomes of VOC, all-cause hospitalisation days, adverse events and serious adverse events, in line with those reported by the phase II RCT on crizanlizumab. ${ }^{18}$ International Society for Pharmacoeconomics and Outcomes Research, Society for Medical Decision (MDM) and UK NICE guidelines were followed in design of the NMA model. ${ }^{27} 29-31$ As the pivotal study on crizanlizumab was conducted within an older adolescent and adult $(\geq 16$ years old) population, the NMA was conducted only on studies that included patients $\geq 16$ years old with SCD. While the pivotal study for L-glutamine (Niihara $e t a l$ ) included patients aged $<16$ years old, a decision was made to include the study to enable a comparison with crizanlizumab. The primary comparison examines the outcomes in the whole population. A sensitivity analysis was subsequently run using the results with Endari in a subgroup of patients aged $>18$ years old (reported in Niihara et al). Evidence networks were generated with nodes corresponding to treatments and edges connecting nodes if at least one RCT comparing corresponding treatments was identified. ${ }^{32}$ An extended network including RCTs with a mixture of child, adolescent and adult populations was investigated for additional direct or indirect evidence on any comparison with crizanlizumab $5.0 \mathrm{mg} / \mathrm{kg}$.

Following NICE guidelines, we employed a shared parameter model for HRs to synthesise studies summarising outcomes in different formats and accounting for differences in trial duration. ${ }^{27}$ Summaries that could be included were total number of events, percentage of patients with events, mean numbers of events, mean or median rates, numbers of patients with at least one event and risk or HR of event. Likelihood and link function for each summary followed MDM and NICE guidelines. ${ }^{2731}$ Total number of events are modelled with a Poisson likelihood and log link, numbers of patients with at least one event are modelled using a Binomial likelihood and complementary log log link, while risk and HRs are modelled on a log scale with a normal likelihood and identify link. In line with NICE recommendations, a Bayesian perspective with vague priors was adopted. ${ }^{27} 31$ Sensitivity to priors was explored with details in online supplemental appendix B; the base case prior has an SD of 100 while the precise prior sensitivity has an SD of 3.16 on $\log$ scale of baseline and treatment effects. Fixed and 
random effect were considered with choice being made on basis of model fit; meta-regressions were also explored to assess heterogeneity due to trial duration, proportion female, mean age, proportion homozygous haemoglobin S (HbSS) genotype, proportion hydroxyurea use and proportion black or African-American. ${ }^{33}$ Different doses of the same drug were analysed independently. If a connected evidence network could be formed using only RCTs, single-arm study evidence was discarded. The reference treatment in all analyses was placebo. If feasible, inconsistency between direct and indirect evidence was planned to be tested by node-splitting and an independent means inconsistency model. ${ }^{19}$ All analyses were conducted using the MCMC software of OpenBUGS V.3.2.3. ${ }^{34}$ Two MCMC chains with 400000 iterations for burn-in and 30000 iterations for posterior sampling were used. Convergence was assessed by visual inspection and the Gelman-Rubin statistic. ${ }^{34} 35$ Further details of the modelling methods are provided in online supplemental appendix E.

We generated HRs with 95\% CrI of high-dose crizanlizumab $5.0 \mathrm{mg} / \mathrm{kg}$ relative to each comparator. We estimated the Bayesian probability that crizanlizumab was superior (lower hazard of event) or inferior (higher hazard of event). These probabilities are the Bayesian equivalent of one-sided $p$ values. In line with the recommendations of the American Statistical Association, we did not adopt a strict threshold for interpreting these Bayesian probabilities, ${ }^{36}$ but instead reported the probability itself. Probabilities are interpreted to suggest evidence in favour of a hypothesis if it lay lower than 5\% or above $95 \%$, and weak evidence if the probability was between $5 \%$ and $10 \%$ or $90 \%$ and $95 \%{ }^{37}$

\section{Patient and public involvement}

Patients and/or the public were not involved in the design, or conduct, or reporting, or dissemination plans of this research.

\section{RESULTS}

\section{SLR results}

We retrieved 3388 records from electronic databases, ClinicalTrials.gov and Sins $e t$ al, 2017. After removing duplicates and irrelevant records, we screened 250 full-text articles. Fifty one studies (67 references) were included to perform evidence evaluation for the NMA (figure 1). Full details and references for the 51 studies are included in online supplemental appendix B. We also identified 14 additional ongoing clinical RCTs or completed RCTs without publication, which investigated effects of nonhydroxyurea treatments on SCD patients. ${ }^{38-51}$

Of 51 studies, duration of follow-up was reported in 41 studies and, among RCTs in the $\geq 16$ years old population, duration ranged from 30 days in Wun et a $\tilde{l}^{22}$ to 52 weeks in Ataga $e t a l{ }^{18}$ This range represents substantial variation in follow-up, but the methods used for NMA model trial follow-up compare annualised hazards in order to adjust for this difference.

The proportion of female patients varied across RCTs, ranging from 0.44 in Glassberg et $a l^{53}$ to 0.60 in Sins $e t a l^{54}$ so qualitatively similar proportions. Across all 51 studies, the proportion of females varied from 0.23 in Gupta $e t a \bar{l}^{5}$ to 1.00 in de Abood et $a l^{56}$ representing a more substantial difference. In the $\geq 16$ years old population RCTs, age ranged from 20.5 years in Pace $e t a \tilde{l}^{7}$ to 35.5 years in Ataga et al..$^{58}$ Across all 51 studies, the mean age ranged from 4.8 years in Adegoke et a $\tilde{p}^{9}$ to 48.8 years in Bridges et $a l^{60}{ }^{60}$ The proportion with HbSS genotype ranged from 0.60 in Wun et $a \tilde{l}^{2}$ to 1.00 in several studies that restricted enrolment to patients with HbSS disease alone, including Ataga $e$ t $a b^{5}$ in the $\geq 16$ years old population. Although HbSS is indicative of absolute outcomes (prognostic factor), there is no known evidence that it is an effect modifier, so the NMA remains feasible. ${ }^{33}$ Proportion of patients reported as black or African American ranged from 0.53 in NCT02482298 ${ }^{61}$ to 1.00 in Styles. ${ }^{62}$ Several studies excluded patients with history of hydroxyurea usage, including Bao et $a l^{63}$ in the $\geq 16$ years old population. In the $\geq 16$ years old population, this otherwise varied from 0.42 in Sins $e t a b^{54}$ to 0.67 in Niihara $e t a l^{12}$ making it somewhat comparable.

\section{Construction of evidence networks}

Of the 51 studies identified, there were 17 non-controlled studies that were excluded from the NMA due to lack of common comparators and potential bias. Of the 34 remaining RCTs, only eight were conducted solely in older adolescent and adult ( $\geq 16$ years old) patients. ${ }^{1852-5457586162}$ As the only RCT identified on L-glutamine, Niihara et $a l^{12}$ was included in the network. This gave nine RCTs in the $\geq 16$ years old population evidence networks. Five of these studies used a VOC definition comparable to that in Ataga et al li $^{18} 575862$ (details in online supplemental appendix E). The only study that examined transfusions was a conference abstract by Vichinsky. As the authors did not specify the definition of VOC or a placebo control, this study was excluded from the NMA. ${ }^{64}$ Online supplemental appendix $\mathrm{F}$ shows the characteristics of included studies in the NMA. Analysed evidence networks are provided in figure 2.

In addition to crizanlizumab $5.0 \mathrm{mg} / \mathrm{kg}$ and $2.5 \mathrm{mg} /$ $\mathrm{kg}$, multiple doses of other drugs were included in the networks. Ticagrelor was studied as both two times per day $45 \mathrm{mg}$ (high-dose) and $10 \mathrm{mg}$ (low-dose ${ }^{61}$; N-acetylcysteine (NAC) as $600 \mathrm{mg}$ (low-dose), $1200 \mathrm{mg}$ (mid-dose) and $2400 \mathrm{mg}$ (high-dose ${ }^{57}$; senicapoc with a loading dose of $20 \mathrm{mg}$ two times per day for 4 days followed by $10 \mathrm{mg}$ daily maintenance ${ }^{62}$ and as a low-dose and high-dose formulation corresponding to single loading doses of $100 \mathrm{mg}$ and $150 \mathrm{mg}$, respectively, and maintenance $6 \mathrm{mg}$ and $10 \mathrm{mg}$ daily, respectively. ${ }^{58}$

Cochrane risk of bias assessment for the nine RCTs included in NMA is reported in full in online supplemental appendix E. Risk of bias was low in all categories 


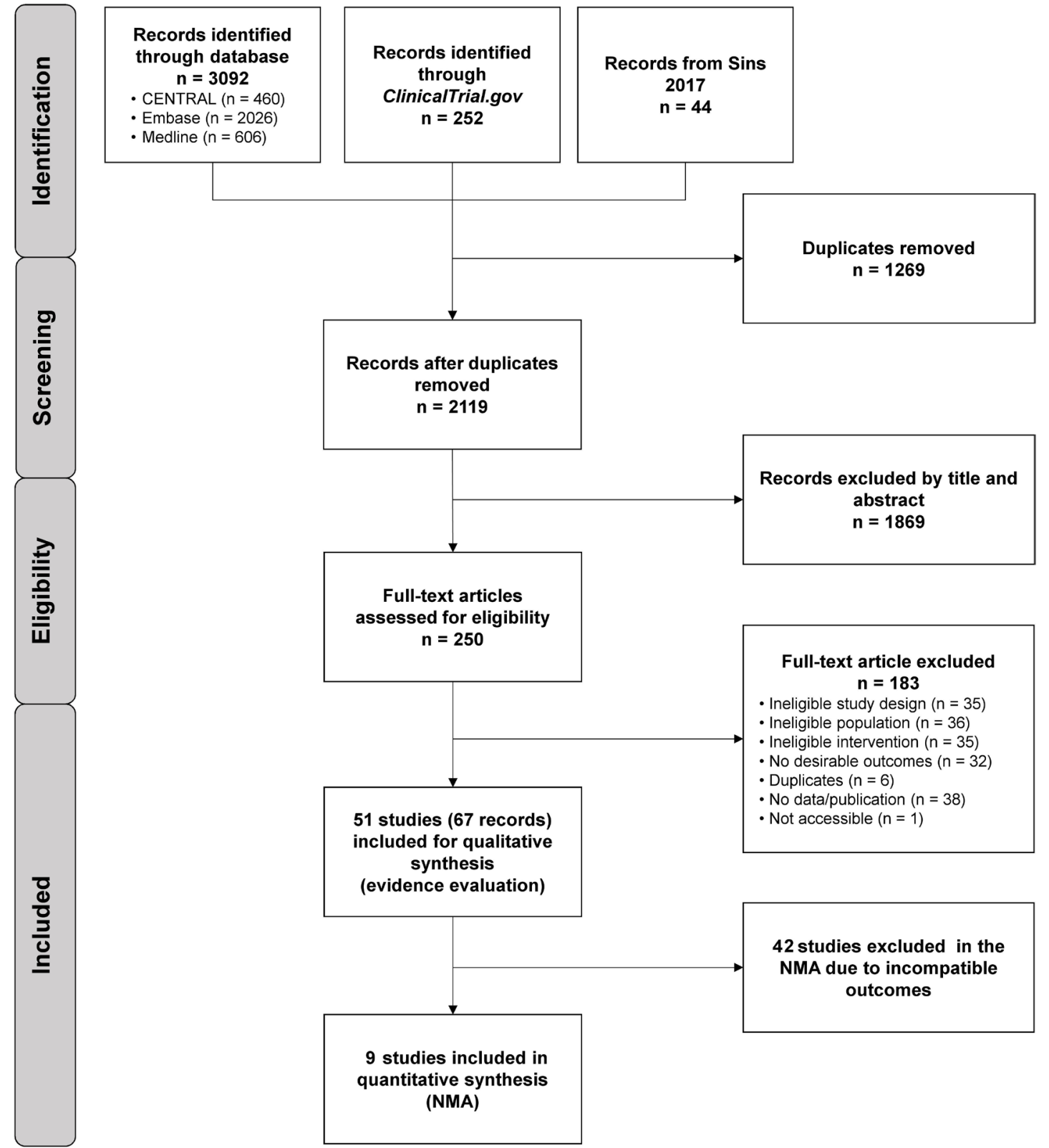

Figure 1 SCD prisma flow chart. NMA, network meta-analysis; SCD, sickle cell disease.

for three of these studies (two studying senicapoc and one mometasone), and was low in all except incomplete outcome data in Ataga $\mathrm{et} \mathrm{al.}{ }^{18}$ Three studies were at unclear risk of bias due to random sequence generation and allocation concealment (studying ticagrelor, L-glutamine and NAC doses). ${ }^{125761}$ Sins et al (studying NAC) was at low risk of bias for all categories except incomplete outcome data, on which it was at high risk of bias. ${ }^{54}$ Wun et al (studying prasugrel) was at unclear risk of bias on random sequence generation, allocation concealment and blinding but low risk of bias on remaining categories. ${ }^{52}$

\section{NMA results}

A fixed effects NMA approach was used for the primary analyses. The NMA models converged well and fit, assessed by comparing residual deviance to total number of data points, was good for all fixed effects analyses. Random effects analyses did not converge as only one RCT was available on each treatment contrast. Meta-regression to explore covariate effects did not reveal evidence of effect medication but convergence was poor for these models. Fit statistics and model assessment details are provided in online supplemental appendix E. Inconsistency could not be tested as there were no treatment contrasts on which both direct and indirect evidence were available. ${ }^{19}$

We discuss in turn the results of the NMA on VOC, all-cause hospitalisation days, adverse events and serious adverse events. Forest plots of HRs with $95 \%$ CI of crizanlizumab versus all comparators are provided in figure 3. Bayesian probabilities that crizanlizumab $5.0 \mathrm{mg} / \mathrm{kg}$ is superior or inferior are also provided in this figure. Pairwise results for all treatment comparisons are provided in online supplemental appendix E. 


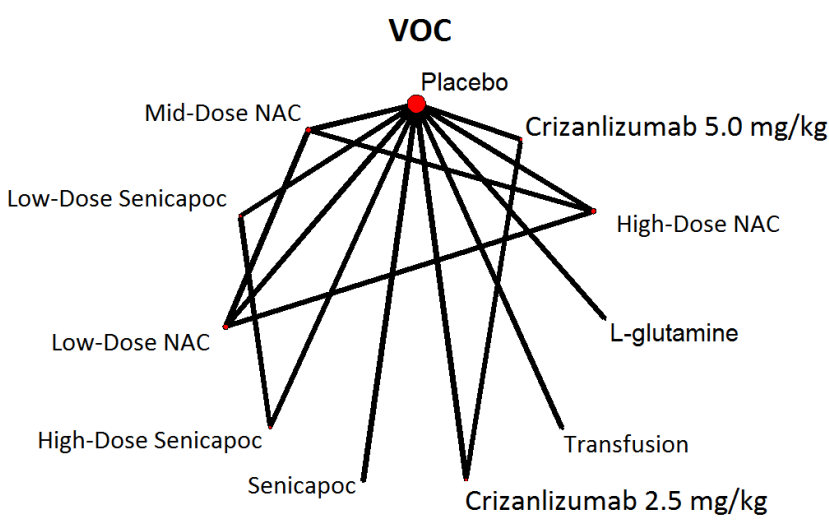

Adverse events

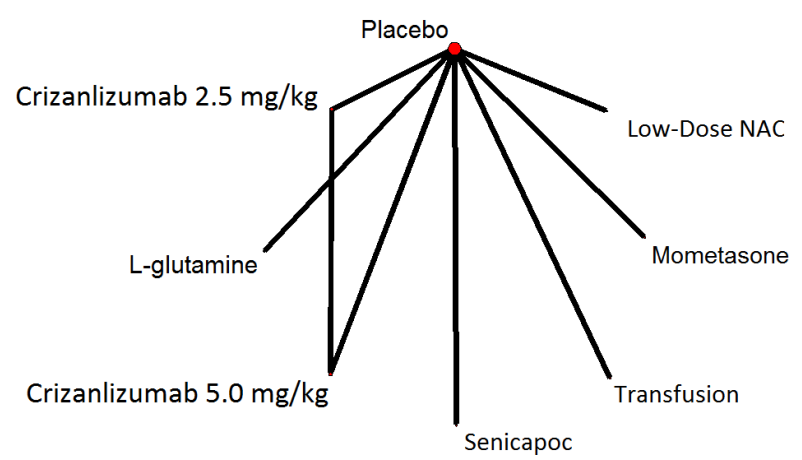

Hospitalization days

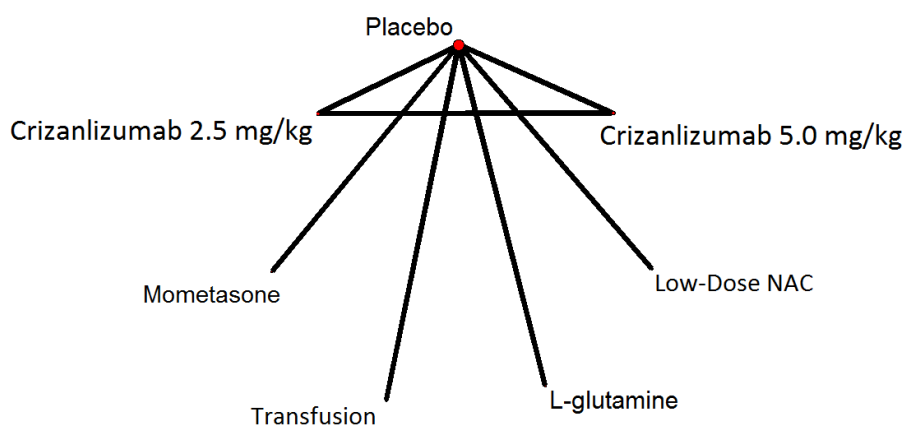

Serious adverse events

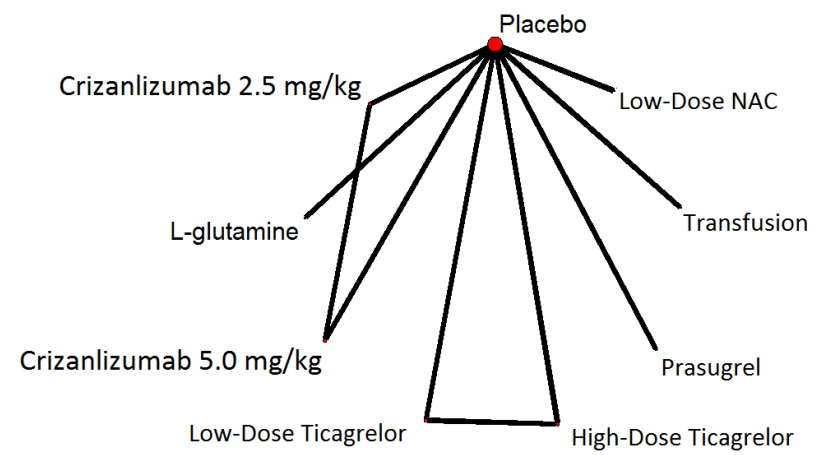

Figure 2 Evidence networks. Each node represents a treatment and nodes are connected by an edge if at least trial has compared the relevant treatments. Any two treatments can be compared if their corresponding nodes can be connected by a path of one or more edges. High-dose crizanlizumab=5 mg/kg 14 times over 52 weeks. Low-dose crizanlizumab=2.5 mg/ kg 14 times over 52 weeks. High-dose ticagrelor=two times per day 45 mg, low-dose ticagrelor=two times per day 10 mg; low-dose $\mathrm{NAC}=\mathrm{N}$-acetylcysteine $600 \mathrm{mg}$, mid-dose $\mathrm{NAC}=\mathrm{N}$-acetylcysteine $1200 \mathrm{mg}$, high-dose NAC=N-acetylcysteine $2400 \mathrm{mg}$; senicapoc=loading dose of $20 \mathrm{mg}$ two times per day for 4 days followed by 10 mg daily maintenance, low-dose senicapoc=single loading dose of $100 \mathrm{mg}$ followed by maintenance $6 \mathrm{mg}$ daily, high-dose senicapoc=single loading dose of $150 \mathrm{mg}$ followed by maintenance $10 \mathrm{mg}$ daily. Five RCTs on crisis=Ataga et al, 2017 (crizanlizumab vs placebo), Niihara et al (Lglutamine vs placebo), Ataga et al, 2011 (senicapoc vs placebo), Ataga et al, 2008 (senicapoc low-dose, senicapoc high-dose vs placebo) and Pace et al (NAC low, mid and high dose vs placebo). Four RCTs on all-cause hospitalisation days=Ataga et al, 2017 (crizanlizumab vs placebo), Niihara et al (L-glutamine vs placebo), Glassberg et al (mometasone vs placebo) and Sins et al (NAC vs placebo). Five RCTs on adverse events=Glassberg et al (mometasone vs placebo), Ataga et al, 2017 (crizanlizumab vs placebo), Ataga et al, 2011 (senicapoc vs placebo), Sins et al (NAC vs placebo) and Niihara et al (L-glutamine vs placebo). Five RCTs on serious adverse events=Ataga et al, 2017 (crizanlizumab vs placebo), Sins et al (NAC vs placebo), Wun et al (prasugrel vs placebo), NCT02482298 (TICAGRELOR vs placebo) and Niihara et al (L-glutamine vs placebo). RCTs, randomised controlled trials; VOC, vaso-occlusive crises.

We found evidence that crizanlizumab $5.0 \mathrm{mg} / \mathrm{kg}$ had a lower hazard of VOC than placebo L-glutamine (HR $0.55,95 \%$ CrI $(0.43,0.69)$; Bayesian probability crizanlizumab $5.0 \mathrm{mg} / \mathrm{kg}$ superior 0.9999$)$, L-glutamine $(0.67$ $(0.51,0.88) ; 0.9982)$ and senicapoc $(0.46(0.32,0.67)$; $>0.9999)$. We found only weak evidence that hazard of VOC was lower on crizanlizumab $5.0 \mathrm{mg} / \mathrm{kg}$ than crizanlizumab $2.5 \mathrm{mg} / \mathrm{kg}(0.81(0.63,1.05) ; 0.9452)$ or low-dose NAC $(0.48(0.18,1.21) ; 0.9396)$. We found no evidence of a difference between crizanlizumab $5.0 \mathrm{mg} / \mathrm{kg}$ and middose NAC $(0.81(0.29,2.18) ; 0.6619)$, high-dose NAC $(1.91(0.57,7.58) ; 0.1507)$, high-dose senicapoc $(0.57$ $(0.15,2.17) ; 0.8010)$ or low-dose senicapoc $(0.53(0.14$, $1.95)$; 0.8334). Results are summarised in table 2. Cumulative ranking plots ('rankograms') are provided in online supplemental appendix B for the interested reader for each of the outcomes of interest. These are plots of the cumulative probability that each treatment is ranked in the top 1, 2, 3 and so on treatments.

In a sensitivity analysis using a rate ratio of 0.64 with $95 \%$ CI $(0.45,0.89)$ in a subgroup of patients aged $>18$ years old reported on page 231 of the publication Niihara et $a l,{ }^{12}$ we found no evidence that crizanlizumab had a lower hazard of VOC than L-glutamine $(0.86(0.57,1.29)$; $0.7707)$. Full results of this analysis are provided in online supplemental appendix E.

We found evidence that crizanlizumab $5.0 \mathrm{mg} / \mathrm{kg}$ had a lower hazard of all-cause hospitalisation days than placebo $(0.58(0.50,0.68) ;>0.9999)$, and crizanlizumab $2.5 \mathrm{mg} /$ $\mathrm{kg}(0.58(0.50,0.68) ;>0.9999)$, but found evidence that hazard was higher than on low-dose NAC (2.08 (1.06, $4.66) ; 0.0166)$. We found weak evidence that hazard of 
VOC

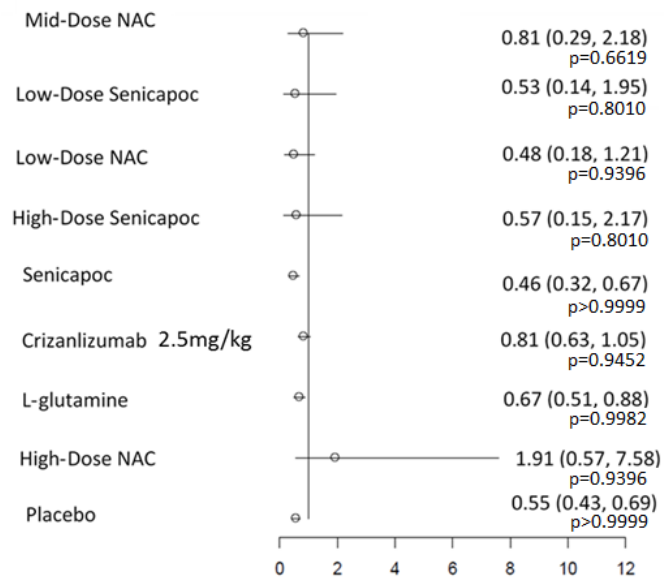

Adverse events

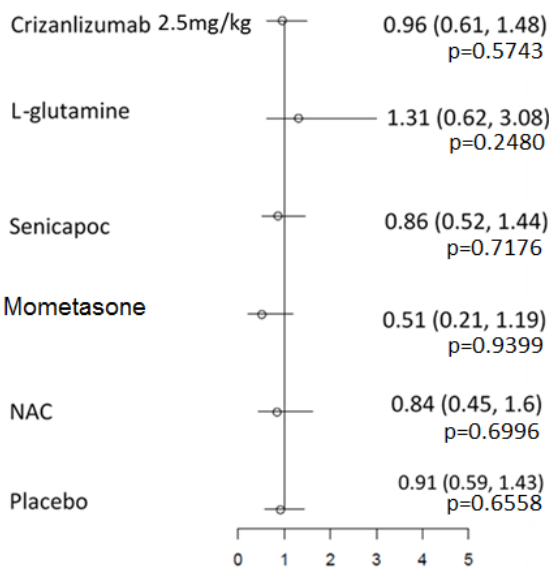

All-cause hospitalization days

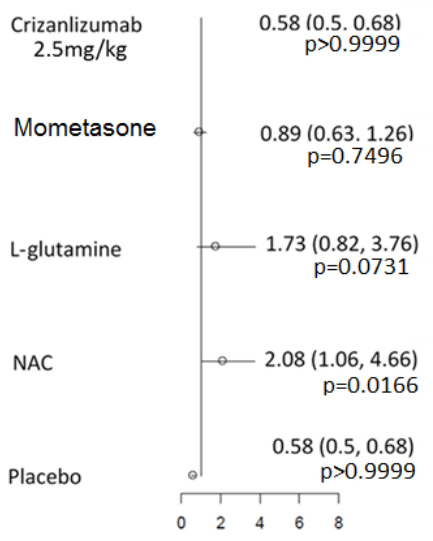

Serious adverse events

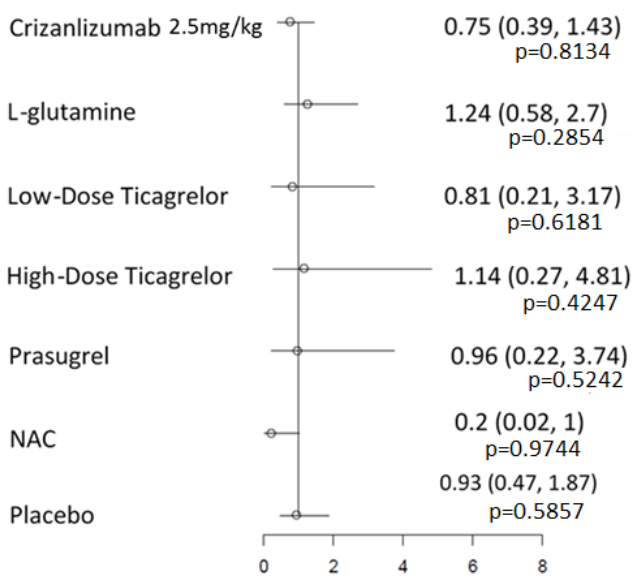

Figure 3 Forest plot. HR $<1$ suggests lower hazard of event on the crizanlizumab. Bayesian probabilities of superiority are proportion of MCMC samples for which crizanlizumab vs comparator HR is above (inferior) or below (superior) 1. High-dose crizanlizumab=5 mg/kg 14 times over 52 weeks. Low-dose crizanlizumab=2.5 mg/kg 14 times over 52 weeks. High-dose ticagrelor=two times per day $45 \mathrm{mg}$, low-dose ticagrelor=two times per day $10 \mathrm{mg}$; low-dose NAC=N-acetylcysteine $600 \mathrm{mg}$, mid-dose NAC=N-acetylcysteine $1200 \mathrm{mg}$, high-dose NAC=N-acetylcysteine $2400 \mathrm{mg}$; senicapoc=loading dose of $20 \mathrm{mg}$ two times per day for 4 days followed by $10 \mathrm{mg}$ daily maintenance, low-dose senicapoc=single loading dose of $100 \mathrm{mg}$ followed by maintenance $6 \mathrm{mg}$ daily, high-dose senicapoc=single loading dose of $150 \mathrm{mg}$ followed by maintenance $10 \mathrm{mg}$ daily. MCMC, Markov chain Monte Carlo; VOC, vaso-occlusive crises.

all-cause hospitalisation days was higher on crizanlizumab $5.0 \mathrm{mg} / \mathrm{kg}$ than on L-glutamine $(1.73(0.82,3.76) ; 0.0731)$ and no evidence of a difference with mometasone $(0.89$ $(0.63,1.26) ; 0.7496)$. Note that all-cause hospitalisation includes admission for VOC but also for adverse events and non-SCD related causes.

The hazard of adverse events-both serious and overall-for crizanlizumab was generally similar or weakly better than other treatments. The exception is that there was weak evidence that crizanlizumab $5.0 \mathrm{mg} / \mathrm{kg}$ had a lower hazard than mometasone $(0.51 \quad(0.21,1.19)$; $0.9399)$. We found no evidence of a difference in hazard of adverse events between crizanlizumab $5.0 \mathrm{mg} / \mathrm{kg}$ and placebo $(0.91 \quad(0.59,1.43) ; 0.6558)$, L-glutamine (1.31 $(0.62,3.08) ; 0.2480)$, crizanlizumab $2.5 \mathrm{mg} / \mathrm{kg} \quad(0.96$ $(0.61,1.48)$; 0.5743), low-dose NAC $(0.84(0.45,1.60)$; $0.6996)$ or senicapoc $(0.86(0.52,1.44) ; 0.7176)$. Similarly, the hazard of serious adverse events on crizanlizumab
$5.0 \mathrm{mg} / \mathrm{kg}$ was lower than on low-dose NAC $(0.20$ (0.02, $1.00) ; 0.9744)$. There was no evidence of a difference on adverse event rates between crizanlizumab $5.0 \mathrm{mg} / \mathrm{kg}$ and placebo (0.93 (0.47, 1.87); 0.5857), L-glutamine (1.24 $(0.58,2.70) ; 0.2854)$, crizanlizumab $2.5 \mathrm{mg} / \mathrm{kg} \quad(0.75$ $(0.39,1.43)$; 0.8134$)$, high-dose ticagrelor (1.14 (0.27, $4.81)$; 0.4247$)$ or low-dose ticagrelor $(0.81$ (0.21, 3.17); $0.6181)$.

Cumulative ranking plots ('rankograms') are provided in online supplemental appendix B for the interested reader. These are plots of the cumulative probability that each treatment is ranked in the top 1, 2, 3 and so on treatments. For crisis, qualitatively, high-dose NAC was most likely to have the top rank (ie, fewest events) rank but was closely followed by crizanlizumab $5.0 \mathrm{mg} / \mathrm{kg}$. For adverse events, L-glutamine had the best (fewest events) rank followed crizanlizumab $5.0 \mathrm{mg} / \mathrm{kg}$ and for serious adverse events, L-glutamine was again best ranked while 
Table 2 Bayesian probabilities that crizanlizumab is superior on each outcome analysed*

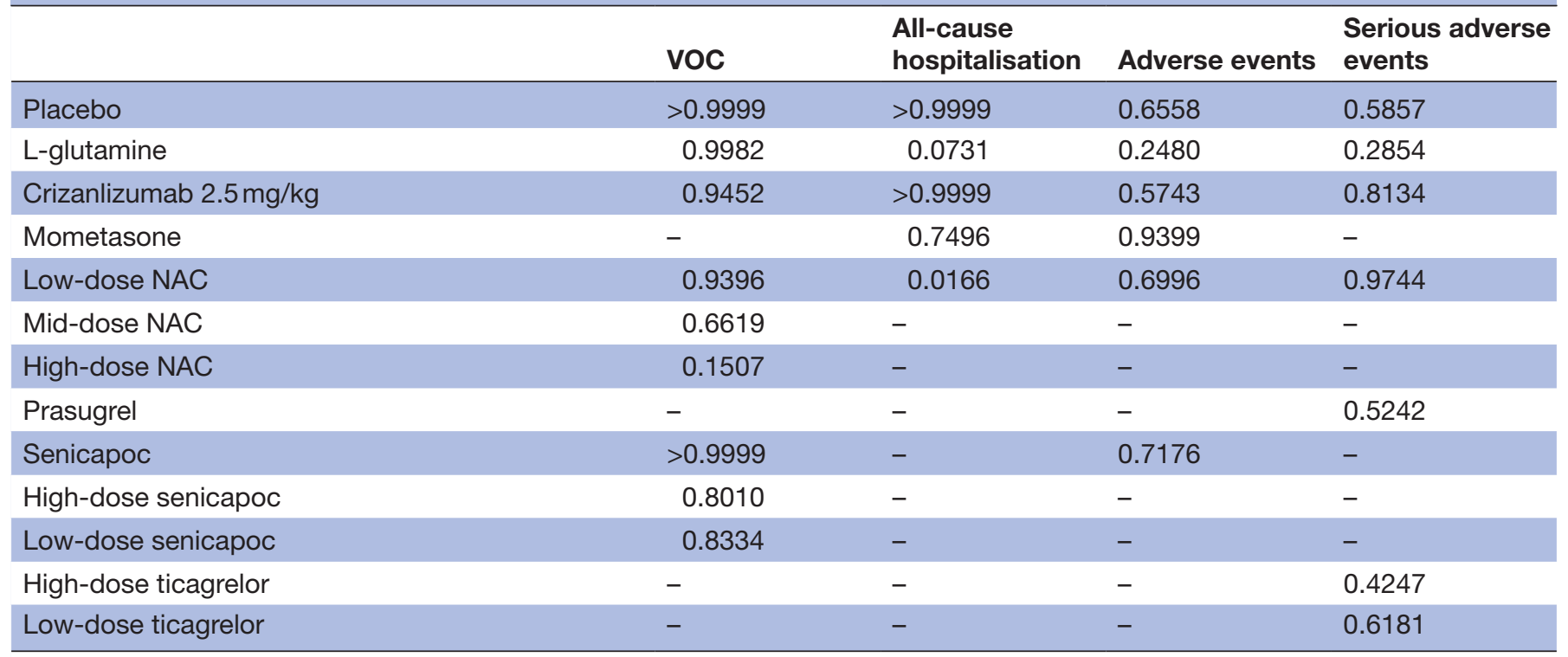

High-dose ticagrelor=two times per day $45 \mathrm{mg}$, low-dose ticagrelor=two times per day $10 \mathrm{mg}$; low-dose NAC=N-acetylcysteine $600 \mathrm{mg}$, middose $\mathrm{NAC}=\mathrm{N}$-acetylcysteine $1200 \mathrm{mg}$, high-dose $\mathrm{NAC}=\mathrm{N}$-acetylcysteine $2400 \mathrm{mg}$; senicapoc=loading dose of $20 \mathrm{mg}$ two times per day for 4 days followed by $10 \mathrm{mg}$ daily maintenance, low-dose senicapoc=single loading dose of $100 \mathrm{mg}$ followed by maintenance $6 \mathrm{mg}$ daily, highdose senicapoc=single loading dose of $150 \mathrm{mg}$ followed by maintenance $10 \mathrm{mg}$ daily.

*Proportion of MCMC samples for which crizanlizumab vs comparator HR is above (inferior) or below (superior) 1. Entry '-' indicates comparator not included in outcome specific evidence network. MCMC, Markov chain Monte Carlo; VOC, vaso-occlusive crises.

crizanlizumab $5.0 \mathrm{mg} / \mathrm{kg}$ was middle ranking. For allcause hospitalisation days, NAC had the best rank (fewest hospitalisations) and was followed by L-glutamine and crizanlizumab $5.0 \mathrm{mg} / \mathrm{kg}$.

A sensitivity analysis assuming more precise priors was conducted and details are provided in online supplemental appendix B. There was little or no impact on results. For example, the HR of VOC for crizanlizumab $5.0 \mathrm{mg} / \mathrm{kg}$ compared with L-glutamine was $(0.67(0.51$, $0.88) ; 0.9982)$ with precise priors and $(0.67(0.51$, $0.88) ; 0.9982)$ in the base case with vague priors. Similarly, the HR of adverse event for crizanlizumab $5.0 \mathrm{mg} /$ $\mathrm{kg}$ compared with L-glutamine was $(1.29(0.62,2.93)$; $0.2480)$ with precise priors and $(1.31(0.62,3.08) ; 0.2480)$ in the base case.

\section{DISCUSSION}

Previous SLRs and meta-analyses of treatments for SCD have demonstrated hydroxyurea to be effective in reducing VOC rates. ${ }^{65}{ }^{66}$ However, patients receiving hydroxyurea therapy can continue to have crises, endorgan damage and a decreased life expectancy. ${ }^{67}$ Crizanlizumab and L-glutamine are promising treatment options for SCD patients not well managed on hydroxyurea, but no direct comparison across these treatments has been conducted. ${ }^{14868}$ Our SLR and NMA are the first looking at the comparative efficacy of new treatments for older adolescent and adult ( $\geq 16$ years old) SCD patients not well managed on hydroxyurea and are therefore of vital importance to this patient population.
Our baseline analysis found that crizanlizumab $5.0 \mathrm{mg} /$ $\mathrm{kg}$ reduced VOC compared with L-glutamine, placebo and senicapoc, and weak evidence of reduction compared with crizanlizumab $2.5 \mathrm{mg} / \mathrm{kg}$ and low-dose NAC. These results, however, were sensitive to whether the L-glutamine efficacy was measured for all patients or only those aged $>18$ years.

We found that crizanlizumab $5.0 \mathrm{mg} / \mathrm{kg}$ reduced allcause hospitalisation days compared with placebo and crizanlizumab $2.5 \mathrm{mg} / \mathrm{kg}$. Conversely, we found evidence that low-dose NAC reduced hospitalisation compared with crizanlizumab $5.0 \mathrm{mg} / \mathrm{kg}$, and weak evidence that L-glutamine reduced hospitalisation compared with crizanlizumab $5.0 \mathrm{mg} / \mathrm{kg}$.

Our analysis found high-dose crizanlizumab $5.0 \mathrm{mg} /$ $\mathrm{kg}$ had a lower hazard of adverse events compared with mometasone and of serious adverse events compared with low-dose NAC. There was no evidence of a difference between $5 \mathrm{mg} / \mathrm{kg}$ crizanlizumab on safety with other treatments.

\section{Strengths}

This SLR was comprehensive in terms of outcomes and interventions and was focused on the target population of crizanlizumab, that of older adolescent and adult $(\geq 16$ years old) SCD patients not well managed, or having failed previous treatment, with hydroxyurea. Our review followed the PRISMA guidelines and checklist. ${ }^{22}$ Risk of bias was assessed using the best practice Cochrane collaboration tool. ${ }^{24}$ To be comprehensive, we searched for both RCT and single-arm evidence but used only RCT evidence 
in the NMA. Our NMA combines direct head-to-head RCT evidence to enable indirect comparisons of interventions (eg, crizanlizumab $5.0 \mathrm{mg} / \mathrm{kg}$ vs L-glutamine) that have not been compared directly; it thus goes beyond the published results of individual studies. Our analysis followed published and international guidelines on indirect comparisons and NMA. ${ }^{27-31}$ On the outcome of VOC, we ensured only studies with a definition compatible with that of the principal crizanlizumab study were analysed. ${ }^{18}$ To include a diverse range of outcome summaries, such as total number of events and numbers of patients with at least one event, a shared parameter Bayesian NMA was employed, as recommended by NICE. ${ }^{27}$

\section{Limitations}

There were several limitations to this SLR and NMA. There was at most only one RCT on each of the treatment contrasts. A similar definition of VOC was used across RCTs but the shared parameter NMA combined RCTs without adjusting for differences in statistical analyses, such as methods for managing drop-outs, used. Differences in RCT follow-up (eg, 30 days in Wun et $a \tilde{l}^{22}$ and 52 weeks in Ataga $e t a l^{18}$ ) limit comparability of annualised hazard rates across treatments. The strength of evidence for comparisons on hospitalisation, adverse events and serious adverse events was weak. Furthermore, we could not include transfusions in the NMA as the only available RCT in an adult population-Vichinsky et $a b^{64}$ - used an unspecified standard of care rather than a placebo control, did not describe the definition of VOC that was used, and was published only as an abstract.

Our NMA model generated results on a HR scale and thus used a complementary log-log link for the binomial likelihood when analysing numbers of patients with at least one event. Although such data could have been modelled using a logit link, and thus generated ORs, this would have made it difficult to link to HR data, or total event data, reported by other studies. However, recent research has found HRs and ORs to be similar in NMA if the numbers of events are low, as they are in our study. ${ }^{69}$

Due to a lack of evidence, the NMA was not able to estimate the relative impact of crizanlizumab treatment on the rate of complicated VOC or organ damage, both of which are important health outcomes for patients and physicians. The heterogeneity variance of random effects models was not identifiable as only one study was available on each contrast. Published informative priors could be considered. ${ }^{70}$ However, the heterogeneity variance would be entirely defined by this prior and its validity would depend on the relevance of a non-SCD clinical area as no NMA has been published previously in SCD. Inconsistency in the network could not be assessed as there were no loops in the evidence networks; it was necessary to assume consistency to enable comparisons with crizanlizumab. As there was no additional indirect evidence to be synthesised with the direct evidence, the NMA does not go beyond individual study results on pairwise comparisons for which there is direct head-to-head evidence (eg, crizanlizumab $5.0 \mathrm{mg} / \mathrm{kg}$ vs placebo). In such cases, individual study results should remain the primary source of comparative data.

A previous SLR in non-hydroxyurea SCD treatments did not conduct quantitative synthesis due to concerns regarding heterogeneity. ${ }^{23}$ Although we considered metaregression on trial duration, proportion female, mean age, proportion HbSS genotype, proportion hydroxyurea use and proportion black or African-American, there was insufficient evidence as there was only one RCT on each treatment contrast. We were also lacking information on the amount of VOCs in the year preceding randomization/treatment start for several of the treatments included in the analysis, a factor known to be prognostic. We therefore had to assume differences in characteristics would not modify treatment effects, even in parameters expected to influence the frequency of VOCs. Although we conducted a sensitivity analysis using results among $>18$ year olds from Niihara et al, that study itself concluded that there was 'no significant interaction between trial group assignment and age'. ${ }^{71}$ On the other hand, if age is an effect modifier, the baseline results should be interpreted cautiously. Future real-world evidence studies may be useful to explore effect modifiers and identify patient types that benefit most from crizanlizumab and other treatments.

Further, caution should be taken when interpreting these results in relation to switching patients from hydroxyurea to crizanlizumab or L-glutamine. Our analysis does not purport to compare crizanlizumab, or indeed L-glutamine or blood transfusions, with hydroxyurea but is instead focused solely on patients who are not well managed on hydroxyurea. Before more evidence is available, physicians should consider treatment with hydroxyurea before consideration of second line treatments. ${ }^{72}$

\section{Conclusion}

Our baseline analysis showed from an SLR and NMA that crizanlizumab reduced crises and hospital days compared with placebo and other treatments with an acceptable adverse event profile in older adolescent and adult $(\geq 16$ years old) SCD patients when compared with other nonhydroxyurea treatments. The VOC results, however, were sensitive to assumptions regarding whether patient age is an effect modifier.

Acknowledgements Systematic literature review was assisted by Luke McGuinness of University of Bristol, Rohan Shirali of Precision Xtract and Emily Glowienka of Precision Xtract. Clifton Chow provided early review of the systematic literature review protocols. We would like to thank Miranda Bailey, Andrew Cavey, Pascal Edrich, Thu Thuy Nguyen and Patrick Urban for their comments and feedback on this manuscript.

Contributors HT drafted the manuscript and conducted and designed and conducted the network meta-analysis. NS ensured medical relevance for the review and analysis and provided context for the results. JJ advised on statistical aspects of the analysis. GJ and JS provided oversight to the whole project. LZ provided project management and administrative support. $\mathrm{H}-\mathrm{YC}$ led the systematic review. $\mathrm{SG}$ validated the network meta-analysis. All authors reviewed and edited the manuscript. 
Funding This work was funded by Novartis Pharma AG, manufacturer of crizanlizumab.

Competing interests Novartis reviewed the manuscript prior to submission. JS and $\mathrm{JJ}$ disclose employment by and equity in by precision HEOR, a consulting firm to the life sciences and broader health care industry. HT discloses personal consulting fees from Novartis Pharma AG, Hoffman La-Roche, Pfizer and Eli Lilly. LZ discloses employment by precision HEOR, a consulting firm to the life sciences and broader health care industry. NS discloses research funding and speaker fees from Novartis Pharmaceutical Company.

Patient consent for publication Not required.

Provenance and peer review Not commissioned; externally peer reviewed.

Data availability statement All data relevant to the study are included in the article or uploaded as supplementary information. All necessary data, coda, and initial values for our OpenBUGS models are provided in the network meta-analysis.

Open access This is an open access article distributed in accordance with the Creative Commons Attribution Non Commercial (CC BY-NC 4.0) license, which permits others to distribute, remix, adapt, build upon this work non-commercially, and license their derivative works on different terms, provided the original work is properly cited, appropriate credit is given, any changes made indicated, and the use is non-commercial. See: http://creativecommons.org/licenses/by-nc/4.0/.

ORCID iD

Lauren Zhao http://orcid.org/0000-0001-6339-149X

\section{REFERENCES}

1 Hassell KL. Population estimates of sickle cell disease in the U.S. Am $J$ Prev Med 2010;38:S512-21.

2 Manwani D, Frenette PS. Vaso-occlusion in sickle cell disease: pathophysiology and novel targeted therapies. Blood 2013;122:3892-8.

3 Zhang D, Xu C, Manwani D, et al. Neutrophils, platelets, and inflammatory pathways at the nexus of sickle cell disease pathophysiology. Blood 2016;127:801-9.

4 Polanowska-Grabowska R, Wallace K, Field JJ, et al. P-SelectinMediated platelet-neutrophil aggregate formation activates neutrophils in mouse and human sickle cell disease. Arterioscler Thromb Vasc Biol 2010;30:2392-9.

5 Frelinger AL, Jakubowski JA, Brooks JK, et al. Platelet activation and inhibition in sickle cell disease (pains) study. Platelets 2014;25:27-35.

6 Sreeramkumar V, Adrover JM, Ballesteros I, et al. Neutrophils scan for activated platelets to initiate inflammation. Science 2014;346:1234-8.

7 Matsui NM, Varki A, Embury SH. Heparin inhibits the flow adhesion of sickle red blood cells to P-selectin. Blood 2002;100:3790-6.

8 Matsui NM, Borsig L, Rosen SD, et al. P-Selectin mediates the adhesion of sickle erythrocytes to the endothelium. Blood 2001;98:1955-62.

9 Wagner DD, Frenette PS. The vessel wall and its interactions. Blood 2008;111:5271-81.

10 Novelli EM, Gladwin MT. Crises in sickle cell disease. Chest 2016;149:1082-93.

11 Lanzkron S. Sickle cell anemia BMJ best practice 2018.

12 Niihara Y, Miller ST, Kanter J, et al. A Phase 3 Trial of I -Glutamine in Sickle Cell Disease. N Engl J Med Overseas Ed 2018;379:226-35.

13 Wang WC, Oyeku SO, Luo Z, et al. Hydroxyurea is associated with lower costs of care of young children with sickle cell anemia. Pediatrics 2013;132:677-83.

14 Hematology ASo. State of sickle cell disease: 2016 report 2016.

15 Vichinsky E, Hoppe CC, Ataga KI, et al. A phase 3 randomized trial of Voxelotor in sickle cell disease. N Engl J Med 2019;381:509-19.

16 Prescribing information for Oxbryta (voxelotor) US food and drug administration, 2019. Available: https://www.accessdata.fda.gov/ drugsatfda_docs/label/2019/213137s000lbl.pdf [Accessed March 11, 2020].

17 Prescribing information for Adakveo (crizanlizumab): US food and drug administration, 2019. Available: https://www.accessdata.fda. gov/drugsatfda_docs/label/2019/761128s000lbl.pdf [Accessed March 11, 2020].

18 Ataga KI, Kutlar A, Kanter J, et al. Crizanlizumab for the prevention of pain crises in sickle cell disease. N Engl J Med 2017;376:429-39.

19 Dias S, Welton NJ, Sutton AJ, et al. Evidence synthesis for decision making 4: inconsistency in networks of evidence based on randomized controlled trials. Med Decis Making 2013;33:641-56.
20 Jansen JP, Naci $\mathrm{H}$. Is network meta-analysis as valid as standard pairwise meta-analysis? it all depends on the distribution of effect modifiers. BMC Med 2013;11:159.

21 Moher D, Liberati A, Tetzlaff J, et al. Preferred reporting items for systematic reviews and meta-analyses: the PRISMA statement. $J$ Clin Epidemiol 2009;62:1006-12.

22 Liberati A, Altman DG, Tetzlaff J, et al. The PRISMA statement for reporting systematic reviews and meta-analyses of studies that evaluate healthcare interventions: explanation and elaboration. $B M J$ 2009;339:b2700.

23 Sins JWR, Mager DJ, Davis SCAT, et al. Pharmacotherapeutical strategies in the prevention of acute, vaso-occlusive pain in sickle cell disease: a systematic review. Blood Adv 2017;1:1598-616.

24 Higgins JPT, Altman DG, Gøtzsche PC, et al. The Cochrane collaboration's tool for assessing risk of bias in randomised trials. BMJ 2011;343:d5928.

25 Wells GS, O'Connell D, Peterson J, et al. The Newcastle-Ottawa scale (NOS) for assessing the quality of nonrandomised studies in meta-analyses, 2013. Available: http://www.ohri.ca/programs/ clinical_epidemiology/oxford.asp [Accessed 1 Oct 2016].

26 Sivia D, Skilling J. Data analysis: a Bayesian tutorial. United States: Oxford University Press, 2006.

27 Dias S, Welton N, Sutton A, et al. Nice dsu technical support document 2: a generalised linear modelling framework for pairwise and network meta-analysis of randomised controlled trials. Report by the Decision Support Unit 2011.

28 Dias S, Ades A, Welton N, et al. Network meta-analysis for decisionmaking: Wiley 2018

29 Jansen JP, Fleurence R, Devine B, et al. Interpreting indirect treatment comparisons and network meta-analysis for healthcare decision making: report of the ISPOR Task force on indirect treatment comparisons good research practices: Part 1. Value Health 2011:14:417-28.

30 Hoaglin DC, Hawkins N, Jansen JP, et al. Conducting indirecttreatment-comparison and network-meta-analysis studies: report of the ISPOR Task force on indirect treatment comparisons good research practices: Part 2. Value Health 2011;14:429-37.

31 Dias S, Sutton AJ, Ades AE, et al. Evidence synthesis for decision making 2: a generalized linear modeling framework for pairwise and network meta-analysis of randomized controlled trials. Med Decis Making 2013;33:607-17.

32 Salanti G, Del Giovane C, Chaimani A, et al. Evaluating the quality of evidence from a network meta-analysis. PLoS One 2014;9:e99682.

33 Dias S, Sutton AJ, Welton NJ, et al. Evidence synthesis for decision making 3: heterogeneity--subgroups, meta-regression, bias, and bias-adjustment. Med Decis Making 2013;33:618-40.

34 Lunn D, Jackson C, Best N, et al. The BUGS book : a practical introduction to Bayesian analysis. Boca Raton ; London: CRC Press, 2013.

35 Gelman A, Rubin DB. Inference from iterative simulation using multiple sequences. Statistical Science 1992;7:457-72.

36 American Statistical Association. American statistical association releases statement on statistical significance and P-VALUES. provides principles to improve the conduct and interpretation of quantitative science March 7 2016;2016.

37 Sterne JA, Davey Smith G. Sifting the evidence-what's wrong with significance tests? BMJ 2001;322:226-31.

38 NCT02179177. apixaban in patients with sickle cell disease 2017

39 NCT02615847. clinical trial to study the safety and tolerability of Memantin Mepha $\otimes^{\circledR}$ in sickle cell disease patients 2017.

40 NCT02594462. contraception in women with sickle cell disease 2018.

41 NCT02380079. dose-escalation study of SCD-101 in sickle cell disease 2018.

42 NCT01702246. Effect of simvastatin treatment on Vaso-occlusive pain in sickle cell disease 2015.

43 NCT01737814. evaluation of purified poloxamer 188 in VasoOcclusive crisis of sickle cell disease (EPIC) 2016.

44 NCT02449616. evaluation of repeat administration of purified poloxamer 1882016.

45 NCT02061202. inhaled mometasone to reduce painful episodes in patients with sickle cell disease 2017

46 NCT02633397. A multi-center study of riociguat in patients with sickle cell diseases 2018.

47 NCT03247218. A Phase - Ila - Ilb, Trial to Study the Safety, Tolerability and Efficacy of Memantine as a Long-term Treatment of SCD 2019.

48 NCT02525107. prevention of Vaso-occlusive painful crisis by using omega-3 fatty acid supplements 2019.

49 NCT01704794. quality of life study for sickle cell patients treated with Jobelyn (Sorghum bicolor extract) 2014. 
50 NCT01202812. A randomized trial of LOVAZA in pediatric sickle cell disease (SCD) 2012.

51 NCT02604368. sickle cell omega-3 treatment trial (SCOT trial) 2020.

52 Wun T, Soulieres D, Frelinger AL, et al. A double-blind, randomized, multicenter phase 2 study of prasugrel versus placebo in adult patients with sickle cell disease. J Hematol Oncol 2013;6:6-17.

53 Glassberg J, Minnitti C, Cromwell C, et al. Inhaled steroids reduce pain and SVCAM levels in individuals with sickle cell disease: a tripleblind, randomized trial. Am J Hematol 2017;92:622-31.

54 Sins JF, Fijnvandraat X; , Dominguez K; , et al. Effects of oral Nacetylcysteine on oxidative stress in patients with sickle cell disease. Blood Conference: 59th annual meeting of the american society of hematology, ASH 2017 United states 2017;130.

55 Gupta VL, Chaubey BS. Efficacy of zinc therapy in prevention of crisis in sickle cell anemia: a double blind, randomized controlled clinical trial. J Assoc Physicians India 1995;43:467-9.

56 MdC deA, Guerrero Z.; Espino F.; et al. R) or Microgynon (R) on the painful crises of sickle cell anemia patients. Contraception 1997;56:313-6.

57 Pace BS, Shartava A, Pack-Mabien A, Mulekar A.; et al. Effects of $\mathrm{N}$-acetylcysteine on dense cell formation in sickle cell disease. $\mathrm{Am} \mathrm{J}$ Hematol 2003;73:26-32.

58 Ataga KI, Smith WR, De Castro LM, et al. Efficacy and safety of the Gardos channel blocker, senicapoc (ICA-17043), in patients with sickle cell anemia. Blood 2008;111:3991-7.

59 Adegoke SA, Shehu UA, Mohammed LO, et al. Influence of lime juice on the severity of sickle cell anemia. $J$ Altern Complement Med 2013;19:588-92.

60 Bridges KRG, B.; Bronte L. A single center experience of GBT440 treatment of severe anemia in sickle cell disease (SCD). Blood Conference: 59th Annual Meeting of the American Society of Hematology, ASH 2017;130.

61 NCT02482298. A study to assess the effect of ticagrelor in reducing the number of days with pain in patients with sickle cell disease. 2016.

62 Ataga KI, Reid M, Ballas SK, et al. Improvements in haemolysis and indicators of erythrocyte survival do not correlate with acute vaso-occlusive crises in patients with sickle cell disease: a phase III randomized, placebo-controlled, double-blind study of the
Gardos channel blocker senicapoc (ICA-17043). Br J Haematol 2011;153:92-104.

63 Bao B, Prasad AS, Beck FWJ, et al. Zinc supplementation decreases oxidative stress, incidence of infection, and generation of inflammatory cytokines in sickle cell disease patients. Trans/ Res 2008;152:67-80.

64 Vichinsky E, Neumayr L, Gold Jl, et al. A randomized trial of the safety and benefit of transfusion vs. standard care in the prevention of sickle cell-related complications in adults: a preliminary report from the phase II NHLBI comprehensive sickle cell centers (CSCC) study of neuropsychological dysfunction and neuroimaging abnormalities in neurologically intact adult patients with sickle cell disease. Blood 2010;116:3221.

65 Lanzkron S, Strouse JJ, Wilson R, et al. Systematic review: hydroxyurea for the treatment of adults with sickle cell disease. Ann Intern Med 2008;148:939-55.

66 Nevitt SJ, Jones AP, Howard J, et al. Hydroxyurea (hydroxycarbamide) for sickle cell disease. Cochrane Database Syst Rev 2017;106.

67 Steinberg $\mathrm{MH}$, Barton F, Castro O, et al. Effect of hydroxyurea on mortality and morbidity in adult sickle cell anemia: risks and benefits up to 9 years of treatment. JAMA 2003;289:1645-51.

68 Niihara YM, Razon S.; Claggett R.; , et al. Phase 3 study of L-glutamine in sickle cell disease: analyses of time to first and second crisis and average cumulative recurrent events. Blood Conference: 59th Annual Meeting of the American Society of Hematology, ASH 2017;130.

69 Thom H, López-López JA, Welton NJ. Shared parameter model for competing risks and different data summaries in meta-analysis: implications for common and rare outcomes. Res Synth Methods 2020;11:91-104.

70 Turner RM, Domínguez-Islas CP, Jackson D, et al. Incorporating external evidence on between-trial heterogeneity in network metaanalysis. Stat Med 2019;38:1321-35.

71 Niihara Y, Miller ST, Kanter J, et al. A phase 3 trial of L-glutamine in sickle cell disease. N Engl J Med 2018;379:226-35.

72 Quinn CT. L-Glutamine for sickle cell anemia: more questions than answers. Blood 2018;132:689-93. 